\title{
Correction to: Aortic adventitial thickness as a marker of aortic atherosclerosis, vascular stiffness, and vessel remodeling in systemic lupus erythematosus
}

\author{
Luis P. Roldan ${ }^{1}$ • Paola C. Roldan ${ }^{2}$ - Wilmer L. Sibbitt $\mathrm{Jr}^{1}$ • Clifford R. Qualls ${ }^{1}$ • Michelle D. Ratliff ${ }^{1}$. Carlos A. Roldan ${ }^{1}$ \\ Published online: 6 November 2020 \\ (C) International League of Associations for Rheumatology (ILAR) 2020
}

\section{Correction to: Clinical Rheumatology https://doi.org/10.1007/s10067-020-05431-7}

In the section of Results of the original published version of the above article the bold text emphasis under the following section should have been deleted.

\section{Clinical and laboratory characteristics of SLE patients and controls}

Patients and controls were similar in age, sex, ethnicity, body mass index, and traditionally defined atherogenic risk factors of arterial hypertension, hyperlipidemia, diabetes mellitus, or smoking (all $p \geq 0.28$ ) (Table 1). However, SLE patients as compared to controls had higher blood pressures and serum levels of glucose and D-dimer and lower values of hemoglobin, platelets, and albumin (all $p \leq 0.05$ ). Patients had a mean age of 29 years at diagnosis of SLE and a mean disease duration of 8 years (Supplemental Table 1). About $71 \%$ of patients had active SLE disease, $91 \%$ had elevated inflammatory markers, and
$57 \%$ had positive antiphospholipid antibodies. With regard to therapy, $39 \%$ were on corticosteroids, $49 \%$ on antimetabolite immunosuppressive, $68 \%$ on hydroxychloroquine or chloroquine, and $41 \%$ on antithrombotic therapy.

\section{Arterial disease in SLE patients as compared to controls}

Values of AT, IMT, and PSEM were higher in SLE patients than in controls (all $p \leq 0.004$ ) even after adjusting for differences between patients and controls for blood pressures, fasting glucose level, hemoglobin level, platelet count, albumin level, and quantitative D-dimer level (all $p \leq 0.01$ ) (Table 2). Also, abnormal aortic AT, atherosclerosis, abnormal stiffness, and overall arterial disease were more frequent in SLE patients than in controls (all $p \leq 0.02$ ).

The remainder of the article remains unchanged.

Publisher's note Springer Nature remains neutral with regard to jurisdictional claims in published maps and institutional affiliations.

The online version of the original article can be found at https://doi.org/ 10.1007/s10067-020-05431-7

Carlos A. Roldan

croldan@salud.unm.edu

1 Department of Medicine, Divisions of Cardiology and Rheumatology, University of New Mexico School of Medicine, Cardiology 5ACC, MSC 10-5550, Albuquerque, NM 87131-0001, USA

2 Division of Cardiology, Oregon Health Sciences Center, Portland, OR, USA 